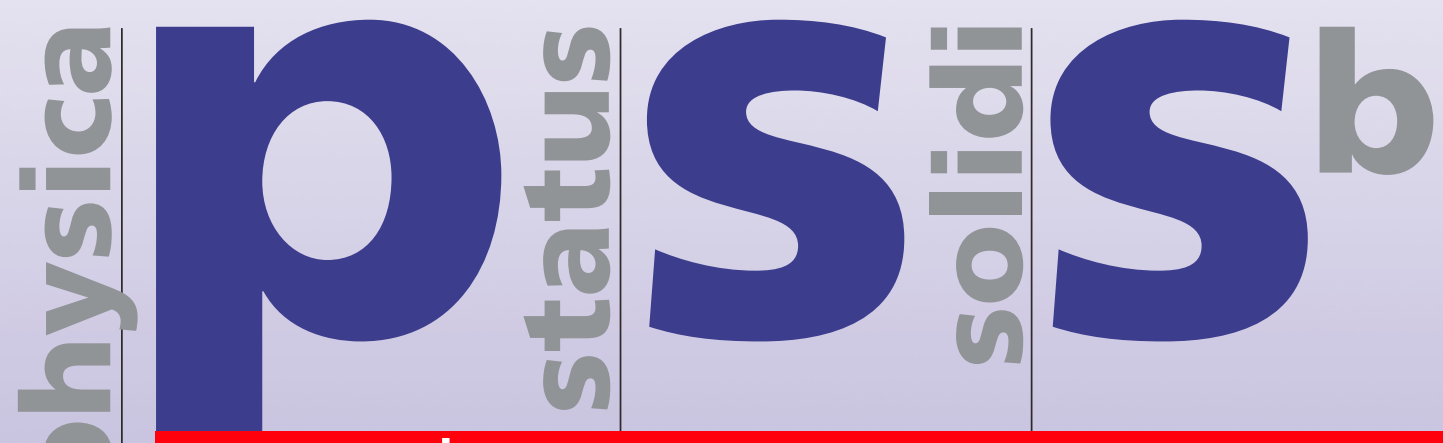

wWw.pss-b.com

basic solid state physics

\title{
Is thermal conductivity time-dependent?
}

\section{Bernd Hüttner}

Institute of Technical Physics, German Aerospace Research Establishment (DLR),

Pfaffenwaldring 38-40, 70569 Stuttgart, Germany

Received 30 April 2008, revised 23 June 2008, accepted 25 June 2008

Published online 22 August 2008

PACS 05.60.Cd, 44.10.+i, 65.40. $-\mathrm{b}$, 72.15.Eb

The question whether thermal conductivity may be time dependent on short time scales is investigated and answered in the affirmative. For this purpose, we make use of a classical relation between the Brownian mean displacement and the thermal conductivity. Interestingly, the transition from the time dependent regime to stationary behaviour is in the vicin- ity of Allen's temperature relaxation time. Calculation of the electronic temperature, when the new time dependent thermal conductivity is used, shows an improvement in the results derived by means of the two-temperature model. Furthermore, the Wiedemann-Franz law is not valid in the time regime investigated.

phys. stat. sol. (b) 245, No. 12, 2786-2790 (2008) / DOI 10.1002/pssb.200844182

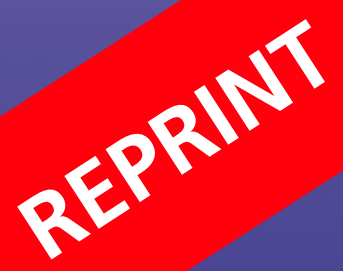




\title{
Is thermal conductivity time-dependent?
}

\author{
Bernd Hüttner ${ }^{*}$ \\ Institute of Technical Physics, German Aerospace Research Establishment (DLR), \\ Pfaffenwaldring 38-40, 70569 Stuttgart, Germany \\ Received 30 April 2008, revised 23 June 2008, accepted 25 June 2008 \\ Published online 22 August 2008 \\ PACS 05.60.Cd, 44.10.+i, 65.40.- b, 72.15.Eb \\ * e-mail bernd.huettner@dlr.de, Phone: +49 7116862 375, Fax: +49 7116862788
}

The question whether thermal conductivity may be time dependent on short time scales is investigated and answered in the affirmative. For this purpose, we make use of a classical relation between the Brownian mean displacement and the thermal conductivity. Interestingly, the transition from the time dependent regime to stationary behaviour is in the vicin-

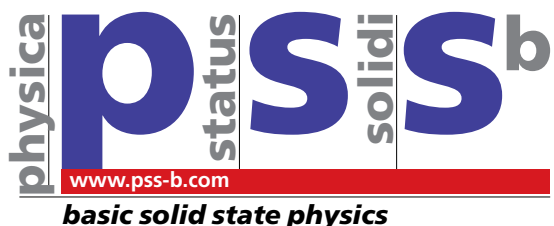

basic solid state physics ity of Allen's temperature relaxation time. Calculation of the electronic temperature, when the new time dependent thermal conductivity is used, shows an improvement in the results derived by means of the two-temperature model. Furthermore, the Wiedemann-Franz law is not valid in the time regime investigated.
1 Introduction At short time scales, a number of unfamiliar phenomena have been observed. For example, electrons and phonons can possess very different temperatures or electrons move in a ballistic way. In this paper, we add a new phenomenon to the list: a time dependence of electronic thermal conductivity. In principle, this is not surprising because from time-dependent electron distribution function, as mentioned below, we can expect also time-dependent transport coefficients. To our knowledge, however, this was not explicit done for the electronic thermal conductivity yet. Moreover, we will provide a rather simple analytical consideration.

Beyond doubt the thermal conductivity is well understood and treated in many textbooks. However, no time dependence is reported but this is also not to expect because measurements are done under steady state condition. The same is true for the theoretical derivation of thermal conductivity that is usually calculated in solid state physics by means of time independent electron distribution function [1]. However, this prerequisite breaks down at short time scales. Below we will show that also the famous Wiedemann-Franz law fails under these conditions. The question whether the thermal conductivity is time dependent or not is important for both the principal understanding and the applications. Deviations from the standard expression of the thermal conductivity are reported under certain circumstances like, for example, the interaction of short laser pulses with metals. It was shown that both the experimental values and the theoretical standard equations are poor approximations of the real behaviour [2]. This is due to the above mentioned existence of different temperatures in the electron and phonon subsystems. Nevertheless, time dependence of thermal conductivity was not considered yet.

2 Theoretical considerations The ballistic behaviour of electrons at short times and the well-known diffusive one at longer times suggests that we have to derive an expression for the transport with such transition. For this purpose, we make use of an analogy between the mean square displacement of a Brownian particle and the thermal diffusivity. Einstein's relation says $\left\langle x^{2}\right\rangle=2 D t$ where the diffusion coefficient $D$ is a constant. The diffusion coefficient may be written also as $D=v^{2} \tau_{d}$ with $v$ as the diffusion velocity and $\tau_{\mathrm{d}}$ as the characteristic time of the underlying diffusion process. The electronic thermal diffusivity is defined in $1 \mathrm{~d}$ by $k_{\mathrm{e}}=\lambda_{\mathrm{e}} / c_{\mathrm{e}}=v_{F}^{2} \tau$ with $\lambda_{\mathrm{e}}$ as the electronic thermal conductivity, $c_{\mathrm{e}}$ as the electronic specific heat, $v_{\mathrm{F}}$ as the Fermi velocity and $\tau$ as the electron scattering time. As expressed already by Einstein, his relation is valid only for times much larger than the characteristic time of the considered process. The generalization to all timescales was first obtained by Ornstein [3] and Fürth [4]. 
Following $[5,6]$, the frequency distribution of the displacement is given in the one dimensional case by

$$
\begin{aligned}
F\left(x_{0}, x, t\right)= & \left(\frac{\beta}{2 \pi D\left(2 \beta t-3+4 \mathrm{e}^{-\beta t}-\mathrm{e}^{-2 \beta t}\right)}\right)^{1 / 2} \\
& \times \exp \left(-\frac{\beta}{D} \frac{\left\{x-x_{0}-v\left(1-\mathrm{e}^{-\beta t}\right) / \beta\right\}^{2}}{\left(2 \beta t-3+4 \mathrm{e}^{-\beta t}-\mathrm{e}^{-2 \beta t}\right)}\right),
\end{aligned}
$$

where $\beta$ is the damping rate and $v$ is the initial velocity. The restriction to one dimension is adapted for the consideration of the interaction of laser radiation with metals which follows because this is in essential a one dimensional problem for not too long times. This is due to the large ratio of the laser spot size to the optical absorption length. From Eq. (1) we get the mean displacement by integration of

$$
\left\langle x^{2}\right\rangle=\int_{-\infty}^{\infty} x^{2} F(x, 0, t) \mathrm{d} x,
$$

where we have set $x_{0}=0$ without loss of generality. When this is done, one finds

$$
\begin{aligned}
\left\langle x^{2}\right\rangle= & 2 D t\left[1-\frac{3}{2 \beta t}+\frac{2}{\beta t} \mathrm{e}^{-\beta t}-\frac{1}{2 \beta t} \mathrm{e}^{-2 \beta t}\right] \\
& +\frac{v^{2}}{\beta^{2}}\left(1-\mathrm{e}^{-\beta t}\right)^{2}
\end{aligned}
$$

This may be rewritten in the final form

$$
\begin{aligned}
\left\langle x^{2}\right\rangle=2 D t[ & 1-\frac{3}{2 \beta t}+\frac{2}{\beta t} \mathrm{e}^{-\beta t}-\frac{1}{2 \beta t} \mathrm{e}^{-2 \beta t} \\
& \left.+\frac{1}{2 \beta t}\left(1-\mathrm{e}^{-\beta t}\right)^{2}\right]=2 t D f(t, \beta),
\end{aligned}
$$

where we have made use of the relation $D=v^{2} \tau_{d}$. The function $f(t, \beta)$ summarizes the expression in the bracket of Eq. (4). With respect to our further application we have to drop the factor 2 in Eq. (4) because only the half space from zero to infinity is involved. This changing is based on the fact that the integrand of Eq. (2) must be even other sides the integral would vanish.

Comparing the simple $1 \mathrm{~d}$ expression of the thermal conductivity of a gas defined by

$$
\lambda=c v^{2} \tau=c D
$$

with the corrected Eq. (4) we obtain for the time dependence of the thermal conductivity

$$
\begin{aligned}
\lambda= & \lambda_{0}(t \gg \tau) \\
& \times\left[1-\frac{3 \tau}{2 t}+\frac{2 \tau}{t} \mathrm{e}^{-\frac{t}{\tau}}-\frac{\tau}{2 t} \mathrm{e}^{-\frac{2 t}{\tau}}+\frac{\tau}{2 t}\left(1-\mathrm{e}^{-\frac{t}{\tau}}\right)^{2}\right],
\end{aligned}
$$

Table 1 Drude scattering times at $T=300 \mathrm{~K}$, times where $f(t, \beta)$ $=0.95$ and temperature relaxation times, respectively, also given is the electronic specific heat and the coefficient of electronphonon energy exchange.

\begin{tabular}{lcllll}
\hline metal & $\tau_{\mathrm{D}}(\mathrm{fs})$ & $\tau_{0.95}(\mathrm{fs})$ & $\tau_{\mathrm{e}}(\mathrm{fs})$ & $c_{\mathrm{e}}\left(\mathrm{J} / \mathrm{cm}^{3} \mathrm{~K}\right)$ & $h_{\mathrm{ex}}\left(\mathrm{GW} / \mathrm{cm}^{3} \mathrm{~K}\right)$ \\
\hline $\mathrm{Ag}$ & 31 & 620 & 571 & 0.02 & 35 \\
$\mathrm{Al}$ & 5.2 & 104 & 109 & 0.038 & 350 \\
$\mathrm{Au}$ & 28 & 560 & 666 & 0.02 & 30 \\
$\mathrm{Cu}$ & 27 & 540 & 583 & 0.028 & 48 \\
$\mathrm{Nb}$ & 4 & 80 & 76 & 0.22 & 2910 \\
\hline
\end{tabular}

where $\lambda_{0}(t \gg \tau)$ is standing for the steady state value and $\beta$ is replaced by $\tau^{-1}$. Clearly, for times much larger than $\tau$ the Eq. (6) becomes time independent. To check the short time limit we expand Eq. (6) when $t / \tau \ll 1$

$$
\begin{aligned}
\lambda & =\lambda_{0}\left[1-\frac{3 \tau}{2 t}+\frac{2 \tau}{t} \mathrm{e}^{-\frac{t}{\tau}}-\frac{\tau}{2 t} \mathrm{e}^{-\frac{2 t}{\tau}}+\frac{\tau}{2 t}\left(1-\mathrm{e}^{-\frac{t}{\tau}}\right)^{2}\right] \\
& \cong \lambda_{0}\left[\frac{1}{2} \frac{t}{\tau}-\frac{1}{6}\left(\frac{t}{\tau}\right)^{2} \cdots\right] .
\end{aligned}
$$

Equation (7) shows the required ballistic behaviour since $\left\langle x^{2}\right\rangle=D \beta t^{2}$ at short times. Note, this comes from the first term of Eq. (7) since an additional time factor results from Eq. (4). By means of Eq. (5) we can directly identify $\tau$ as the electron scattering time.

The function $f(t, \beta)$ is plotted in Fig. 1 for two typical scattering times (Al: 5.2 fs, Ag: 31 fs). Furthermore, we have also added the values of the temperature relaxation time (Al: 109 fs, Ag: $571 \mathrm{fs}$ ) calculated by means of Allen's formula [7]

$$
\tau_{\mathrm{e}}=\frac{c_{\mathrm{e}}}{h_{\mathrm{ex}}}
$$

where $c_{\mathrm{e}}$ is the electronic specific heat and $h_{\mathrm{ex}}$ is the heat exchange coefficient. More details are presented in Table 1.

An interesting point is the saturation of $f(t, \beta)$ in the vicinity of the temperature relaxation time although it depends only on the scattering time. Since niobium shows a rather large scattering in the experimental data for the heat exchange coefficient, from $1700 \mathrm{GW} / \mathrm{cm}^{2}$ [8] up to $4120 \mathrm{GW} / \mathrm{cm}^{2}$ [9], we used for the calculation the average value.

The physical understanding is provided by the nature of the heat exchange coefficient. In essential it consist of three parts the electronic specific heat, the mean square of the phonon frequencies and the electron-phonon coupling constant. If a metal possesses a large heat exchange coefficient the interaction between the electrons and phonons is strong and, therefore; the scattering time and the electron temperature relaxation time are small compared to a metal with a weaker coupling. In fact, the ratio of the temperature relaxation time to the electron scattering time is in all cases about 20 . 


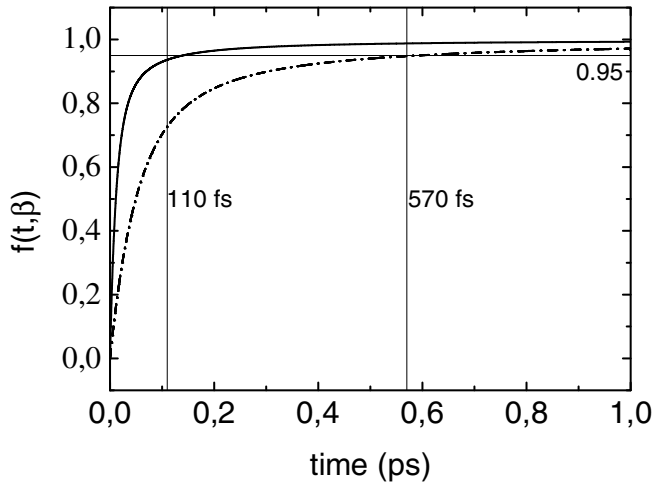

Figure 1 Function $f(t, \beta)$ for $\tau_{\mathrm{D}}=5.2$ fs (full curve, $\mathrm{Al}$ ) and $\tau_{\mathrm{D}}=31 \mathrm{fs}$ (dashed-dotted curve, $\mathrm{Ag}$ ). The vertical lines represent the rounded values of $\tau_{\mathrm{e}}=110 \mathrm{fs}(\mathrm{Al})$ and $\tau_{\mathrm{e}}=570 \mathrm{fs}(\mathrm{Ag})$. The horizontal line corresponds to $f(t, \beta)=0.95$ taken as the onset of saturation.

Let us come now to the question if the crossing of the function $f(t, \beta)$ is accidentally or not close to the temperature relaxation time. It is well known that Fourier's law fails at short time scales [10]. A more suited equation was ad-hoc proposed by Cattaneo [11] and Vernotte [12] independent from each other. It reads

$$
\tau_{\mathrm{e}} \frac{\partial \boldsymbol{q}}{\partial t}+\boldsymbol{q}=-\lambda \nabla T
$$

where we used already for the relaxation time of the heat flow $\tau_{\mathrm{e}}$ as proved in [13]. An illustrative example to derive Eq. (9) is to assume a non-local relation between the heat flow and the gradient of temperature, e.g.,

$$
\boldsymbol{q}(t)=-\int_{-\infty}^{t} \lambda\left(t-t^{\prime}\right) \nabla T\left(t^{\prime}\right) \mathrm{d} t^{\prime}
$$

If the memory kernel is defined as

$$
\lambda\left(t-t^{\prime}\right)=\lambda \frac{1}{\tau_{\mathrm{e}}} \exp \left(-\frac{t-t^{\prime}}{\tau_{\mathrm{e}}}\right),
$$

Eq. (9) is found immediately by differentiation with respect to the upper bound of the integral. It should be noted that a sophisticated derivation of Eq. (9) is provided in the frame of the extended thermodynamics [14] or under use of Boltzmann's equation [13]. Figure 2 shows for silver a log-log-plot of the time-integral of Eq. (11) as $F(t, \alpha)$ with $\alpha=\tau_{\mathrm{e}}^{-1}$ together with the function $f(t, \beta)$.

From the qualitative similarity of both curves in the ballistic range and from the fact that $F(t, \alpha)$ is only determined by $\tau_{\mathrm{e}}$ we can assume that some scattering events are necessary for the transition of the ballistic to the diffusive behaviour. This conclusion is also supported by experiments [15] where the authors observed that the electrons in thin gold films behave ballistic up to about 350 fs after excitation with a femtosecond laser pulse. Consequently, at least a part of the particles travel without scattering across

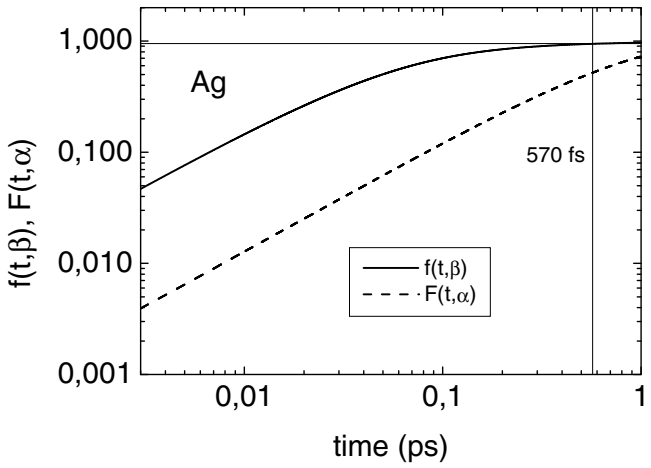

Figure 2 Time dependence of the functions $F(t, \alpha)$ and $f(t, \beta)$; for details see text.

the film due to a subtle balance between electron-electron and electron-phonon scattering as discussed in [15]. Furthermore, it is remarkable that a linear decrease of the effective thermal conductivity with increasing frequency (decreasing time) was shown by molecular dynamic technique and the fluctuation-dissipation theorem in silicon [16] and in a more general consideration in [17].

Now we will investigate what are the consequences of a thermal conductivity predicted by Eq. (6). For this purpose, we have computed the surface electron temperature of $\mathrm{Al}$ and $\mathrm{Ag}$ by means of the two-temperature model (TTM) [18] and the extended two-temperature model (ETTM) [19]. The temperature dependent part of the thermal conductivity was taken as [2]

$$
\lambda_{\mathrm{e}}=\lambda_{\mathrm{e} 0} \frac{T_{\mathrm{e}}}{T_{\mathrm{ph}}\left(1+z\left(T_{\mathrm{e}}, T_{\mathrm{ph}}\right)\right)},
$$

where $z\left(T_{\mathrm{e}}, T_{\mathrm{ph}}\right)$ is defined by the ratio of electron-phonon scattering time to the temperature part of the electronelectron scattering time

$$
z\left(T_{\mathrm{e}}, T_{\mathrm{ph}}\right)=\frac{\tau_{\mathrm{ph}}\left(T_{\mathrm{ph}}\right)}{\tau_{\mathrm{T}}\left(T_{\mathrm{e}}\right)}=4 \pi^{2} B T_{e}^{2}(\mathrm{eV}) \tau_{D}\left(T_{\mathrm{ph}}\right)
$$

with $B$ as an experimental parameter [20] and $\lambda_{\mathrm{e} 0}$ as the steady state value. The TTM was evaluated with $\lambda_{\mathrm{e}}$ from Eq. (12) and with this expression multiplied by the function $f(t, \beta)$ from Eq. (4). The results for the three models are compared for $\mathrm{Al}$ in Fig. 3 and for Ag in Fig. 4. For the time function of the laser intensity we used $\sin ^{2}\left(\pi t / 2 \tau_{\mathrm{L}}\right) H\left(1-t / 2 \tau_{\mathrm{L}}\right)$ where $H(x)$ is the Heaviside step function. In the ETTM only Eq. (12) was applied because this approach is already inherently ballistic.

How could we read these curves? Not unexpected the TTM with the standard thermal conductivity shows the lowest temperature maxima. This is caused by a wellknown and serious fault of the parabolic heat conduction equation namely the infinite heat velocity. In the ETTM a characteristic time, $\tau_{\mathrm{e}}$, is needed to build up the heat flow because a temperature gradient cannot switch on immediately. Consequently, more energy is stored below the sur- 


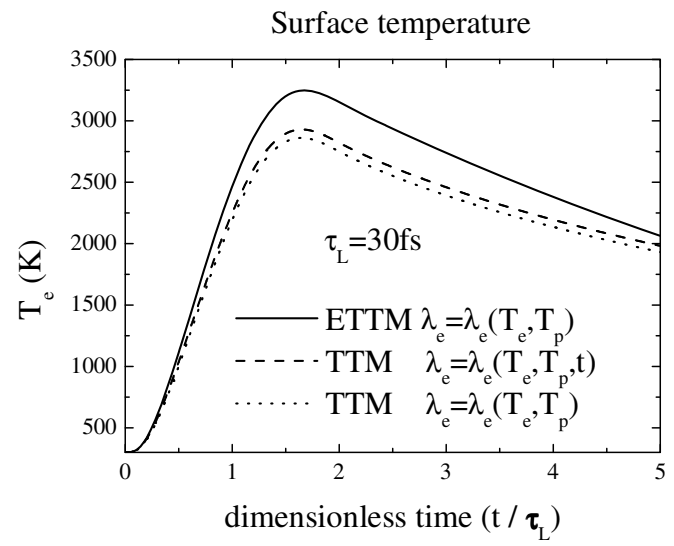

Figure 3 Electronic surface temperature of $\mathrm{Al}$ evaluated with the TTM and ETTM. Laser data: absorbed intensity $I=42 \mathrm{GW} / \mathrm{cm}^{2}$, $\tau_{\mathrm{L}}=30 \mathrm{fs}(\mathrm{FWHM})$, spatial top-hat.

face leading to higher temperatures compared to the TTM. If we take into account a time dependent thermal conductivity in the TTM the evaluated curves are between the two other cases. At short times, the conductivity is low leading to an effect similar as in the ETTM. With increasing time, the influence of the infinite heat velocity becomes more important. Therefore, for small values of $\tau_{\mathrm{e}}$ the curves of the two TTM models are closer since only for a very short time the thermal conductivity is clearly below its stationary value but with increasing $\tau_{\mathrm{e}}$ the curves of the TTM with $\lambda_{\mathrm{e}}=\lambda_{\mathrm{e}}(t)$ become more like to the ETTM ones.

For a more modern treatment of this subject, the appropriated formalism is provided by the quantum kinetic theory.

For example, one could start from the non-Markovian quantum Landau equation [21] but it would be a cumbersome task far beyond the scope of this paper. However, we will provide a qualitative consideration with the goal to find a deeper understanding of the phenomenon. It is well known that the Wiedemann-Franz law fails if inelastic scattering is present [1].

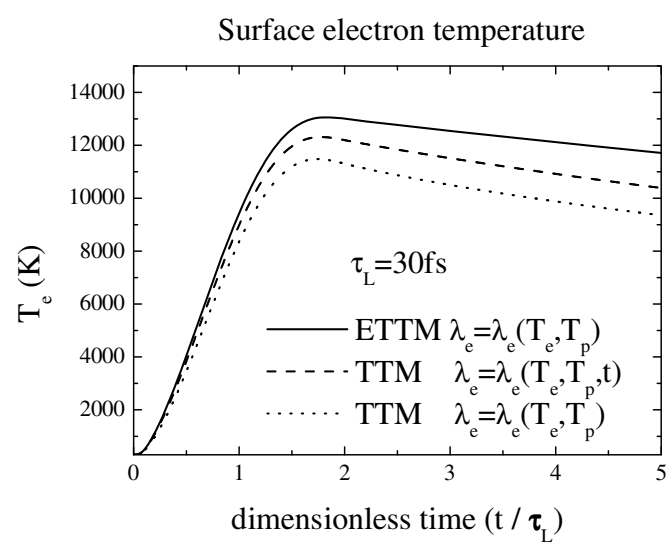

Figure 4 Electronic surface temperature of Ag evaluated with the TTM and ETTM. Laser data: absorbed intensity $I=20 \mathrm{GW} / \mathrm{cm}^{2}$, $\tau_{\mathrm{L}}=30 \mathrm{fs}(\mathrm{FWHM})$, spatial top-hat.
To guarantee that the scattering is elastic it is enough to demand that its probability can be written as

$$
W_{\boldsymbol{k} \boldsymbol{k}^{\prime}} \sim \delta\left(E(\boldsymbol{k})-E\left(\boldsymbol{k}^{\prime}\right)\right)\left|\left\langle\boldsymbol{k}|V| \boldsymbol{k}^{\prime}\right\rangle\right|^{2}
$$

where $V$ is the interaction potential. Due to the energy delta function the energy of the electron is conserved in each collision. In the quantum kinetic theory, the energy delta function is replaced by $\cos \left\{\left(E-E^{\prime}\right) t / \hbar\right\}$ leading to an energy broadening. This changed expression is related to the finite collision duration. On short time scales, $t<\tau$, this effect is especially important since it allows scattering events which do not conserve the electron energy. Based on this statement we can conclude that on short time scales the Wiedemann-Franz law is not obeyed. From Fig. 1, we estimate the time range of violation from zero up to around $\tau_{\mathrm{e}}$. Another support for this proposition was given in [13] where was shown that the thermal current relaxes with $\tau_{\mathrm{e}}$ but the electrical current with the Drude scattering time $\tau_{\mathrm{D}}$.

3 Conclusions In this paper, we have shown that the thermal conductivity is time dependent on short time scales. For this purpose, we stressed a classical analogy between Brownian motion and thermal conductivity. We find out that the conductivity increases rapidly from zero to its stationary value. It is interesting that the time needed for saturation, taken as $f(t, \beta)=0.95$, is in rather good agreement with the temperature relaxation time. Roughly speaking, at times smaller than the temperature relaxation time the electrons possesses a ballistic behaviour and a diffusive one for times larger than $\tau_{\mathrm{e}}$ in agreement with the experimental evidence [15]. Furthermore, by calculating the electronic surface temperature we deduced that the use of Eq. (6) could correct, at least partly, the effect of the incorrect infinite thermal speed inherent in the TTM. Moreover, with the same argument as that used to explain the failure of the Wiedemann-Franz law in the case of inelastic scattering we stated that this law is also not valid in the relevant time range.

\section{References}

[1] N. W. Ashcroft and N. D. Mermin, Solid State Physics (Saunders, Philadelphia, PA, 1976), pp. 322-323.

[2] B. Hüttner, J. Phys.: Condens. Matter 10, 6121 (1998).

[3] L. S. Ornstein, Proc. Amst. 21, 96 (1919).

[4] R. Fürth, Z. Phys. 2, 244 (1920).

[5] G. E. Uhlenbeck and L. S. Ornstein, Phys. Rev. 36, 823 (1930).

[6] S. Chandrasekhar, Rev. Mod. Phys. 15, 1 (1943).

[7] P. B. Allen, Phys. Rev. Lett. 59, 1460 (1987).

[8] K. M. Yoo, X. M. Zhao, M. Siddique, R. R. Alfano, D. P. Osterman, M. Radparvar, and J. Cunniff, Appl. Phys. Lett. 56, 1908 (1990).

[9] S. D. Brorson, A. Kazeroonian, J. S. Moodera, D. W. Face, T. K. Cheng, E. P. Ippen, M. S. Dresselhaus, and G. Dresselhaus, Phys. Rev. Lett. 64, 2172 (1990).

[10] V. C. Liu, J. Non-Equilib. Thermodyn. 4, 143 (1979). 
[11] C. A. Cattaneo, C. R. Acad. Sci. 247, 431 (1958).

[12] P. Vernotte, C. R. Acad. Sci. 246, 3154 (1958).

[13] B. Hüttner, J. Phys.: Condens. Matter 11, 6757 (1999).

[14] D. Jou, J. Casas-Vazquez, and G. Lebon, Rep. Prog. Phys. 62, 1035 (1999).

[15] C. Suarez, W. E. Bron, and T. Juhasz, Phys. Rev. Lett. 75 4536 (1995).

[16] S. G. Volz, Phys. Rev. Lett. 87, 74301 (2001).
[17] B. S. Shastry, Phys. Rev. B 73, 0815177 (2006)

[18] S. I. Anisimov, B. L. Kapeliovich, and T. L. Perel'man, Sov. Phys.-JETP 39, 945 (1974 ).

[19] B. Hüttner, and G. Rohr, Appl. Surf. Sci. 126, 129 (1996).

[20] G. R. Parkins, W. E. Lawrence, and R. W. Christy, Phys. Rev. B 23, 6408 (1981).

[21] M. Bonitz, Quantum Kinetic Theory (B. G. Teubner, Stuttgart, Leipzig, 1998), p. 140 